\title{
Hemorrhagic Cytomegalovirus Colitis in a Postoperative Colon Cancer Patient
}

\author{
Motonobu Saito $^{a, c}$ Atsushi Ishino $^{b}$ Taisuke Ito $^{a}$ Takeshi Sakuma $^{a}$ \\ Masami Matsuzaki ${ }^{a}$ Naoto Katagata $^{a}$ Fumiaki Watanabe ${ }^{a}$ \\ Seiichi Takenoshita ${ }^{c}$ Tadashi Nomizu $^{a}$ \\ Departments of ${ }^{a}$ Surgery and ${ }^{b}$ Gastroenterology, Hoshi General Hospital, Koriyama, and \\ ${ }^{c}$ Department of Organ Regulatory Surgery, Fukushima Medical University School of \\ Medicine, Fukushima, Japan
}

\section{Key Words}

Cytomegalovirus $\cdot$ Immunocompromised host $\cdot$ Hemorrhagic colitis

\begin{abstract}
We report a case of hemorrhagic cytomegalovirus (CMV) colitis, occurring in a postoperative patient due to a weakened immune system. An 85-year-old woman with a medical history, including chronic renal failure treated with oral administration of prednisolone, underwent colectomy due to an ascending colon cancer. While the postoperative course was favorable, she exhibited acute severe abdominal pain and massive bloody discharge after 11 days of surgery. Her colonoscopic examination showed multiple longitudinal ulcers on the anastomosis. In addition to these endoscopic findings, her past medical history helped suggest $\mathrm{CMV}$ colitis. Because serological testing revealed positive CMV antigen, she was finally given a diagnosis of CMV colitis and received intravenous ganciclovir for the initial treatment. Hemorrhagic CMV colitis after colectomy is an important postoperative complication; we therefore present our case with diagnosis and treatment experience.
\end{abstract}

\section{Introduction}

Cytomegalovirus (CMV) colitis is common among immunocompromised patients who are more prone to opportunistic infections. After the infection of CMV is detected by histology or serology, antiviral treatment is usually chosen initially. Since CMV disease cannot be diagnosed on clinical symptoms alone, diagnosis and treatment are sometimes 
delayed. Here, we report a case of a hemorrhagic CMV colitis that occurred in an immunocompromised patient after a colectomy due to colon cancer and was successfully treated with ganciclovir.

\section{Case Report}

An 85-year-old woman underwent esophagogastroduodenoscopy and colonoscopy for investigation of anemia. A laterally spreading tumor of the granular type, $85 \mathrm{~mm}$ in diameter, was detected at the cecum (fig. 1). Although magnifying image-enhanced colonoscopy suggested a tubulovillous adenoma, surgical resection, not endoscopic mucosal resection, was chosen because of the size of the tumor. However, as she had a medical history including breast cancer, myocardial infarction, general edema due to chronic renal failure (oral administration of prednisolone $5 \mathrm{mg}$ /day), diverticulosis of sigmoid colon, and bilateral ureteral stent placement due to retroperitoneal fibrosis, she did not want to take medical treatment anymore but to monitor her own condition for a while. After 3 months, she finally agreed to have surgery and was admitted to our hospital.

The patient underwent ileocecal resection followed by stapled functional end-to-end anastomosis (fig. 2). During the perioperative period, steroid was administered by intravenous infusion. The postoperative course was favorable; dietary intake and oral drug administration were started on postoperative day 4 and both flatus and stool passed. However, she had sudden abdominal pain and heavy bloody discharge on postoperative day 11. Although conservative treatment, including blood transfusion therapy for anemia, was performed initially, there was no improvement after a week. We then consulted the physician and decided to investigate the digestive tract. Colonoscopy revealed bleeding and the longitudinal ulcer at the anastomosis (fig. $3 \mathrm{a}, \mathrm{b}$ ). In addition to the endoscopic findings, the patent's immunocompromised state suggested possible CMV colitis. Because CMV antigen was confirmed in peripheral blood samples, intravenous antiviral treatment was started from the postoperative day 23 (ganciclovir, $150 \mathrm{mg} /$ day). After 4 days of the treatment, bloody discharge stopped, and at 8 days, improvement of abdominal pain was observed. The antiviral treatment was continued for 2 weeks until the serological confirmation of negative CMV antigen. After improvement of the colitis-related symptoms, the patient was discharged from our hospital.

\section{Discussion}

CMV infection often develops a latent infection with no signs or symptoms after acute infection [1]. Although most people are exposed to CMV in their lifetime, typically only the patients with weakened immune systems, such as AIDS, malignancy, organ transplant or bone marrow transplant, and steroid or immunosuppressive treatments, become ill by reactivating CMV and present with CMV pneumonia, gastroenteritis, retinitis, and encephalitis. CMV disease is often diagnosed by pathologic and serologic confirmation because clinical symptoms are not specific [2]. CMV colitis usually presents with abdominal pain, diarrhea, hematochezia, and fever. Furthermore, colonoscopic findings of CMV colitis mimic many conditions, including pseudomembranous colitis, ischemic colitis, ulcerative colitis and Crohn's disease [3]. In our case, as we initially considered the bleeding from the diverticulum or anastomosis, conservative treatment was chosen. After one week of conservative therapy, there was no sign of improvement in bloody discharge; therefore, we decided to perform 
Saito et al.: Hemorrhagic Cytomegalovirus Colitis in a Postoperative Colon Cancer Patient

colonoscopy. We were hesitant to perform colonoscopy due to our patient having had a colectomy.

According to the meta-analysis of CMV colitis in immunocompetent hosts, the highest mortality rates were associated with immune modulating conditions that include 16 patients [4]. Of those analyzed, 5 patients (31.3\%) received colectomy as initial treatment and 8 patients $(50 \%)$ and 6 patients $(37.5 \%)$ had coexisting renal failure and diabetes mellitus, respectively [4]. In our case, as the patient was given steroids due to chronic renal failure and the colonoscopy revealed the longitudinal ulcer at the anastomosis, we were able to suspect CMV colitis and confirmed the diagnosis serologically. Because endoscopic biopsy was not performed, CMV infection was not evaluated histologically. Of note, we performed immunohistochemistry of CMV protein using resected colon cancer specimen, though CMVpositive stain was not detected. This result seems to relate with the fact that CMV is not associated with carcinogenesis and reactivation of latent CMV infection causes CMV colitis $[5,6]$.

The first choice of treatment of CMV infection is antiviral therapy [7, 8]. Systemic antiviral treatment has provided significant advances and has resulted in dramatically improved outcomes [7, 8]. Treatment time usually ranges from 1 to 4 weeks, depending on the treatment effect such as improvement of the symptoms and/or confirmation of the negative CMV antigen. In our case, despite the necessity of reducing the dose of ganciclovir due to renal failure, the patient showed good response to systemic therapy and did not require further treatment. Fortunately, as she did not show uncontrollable heavy bleeding or symptoms of bowel perforation, surgical resection was not needed.

The case we mentioned above can potentially occur in immunocompromised patients. With recent progress in medicine, the number of high-risk patients who undergo surgery is increasing; therefore, the reactivation of latent CMV infection in immunocompromised patients should be actively considered for the differential diagnosis, leading to timely diagnosis and appropriate treatment.

\section{Acknowledgements}

We thank Dr. Yoshihiro Nozawa for histological advice.

\section{Disclosure Statement}

Motonobu Saito and the other co-authors have no conflict of interest.

\section{References}

1 Vancikova Z, Dvorak P: Cytomegalovirus infection in immunocompetent and immunocompromised individuals - a review. Curr Drug Targets Immune Endocr Metabol Disord 2001;1:179-187.

2 de la Hoz RE, Stephens G, Sherlock C: Diagnosis and treatment approaches of CMV infections in adult patients. J Clin Virol 2002;2(25 suppl):S1-S12.

3 Bloomfeld RS: Are we missing CMV infections in patients hospitalized with severe colitis? Inflamm Bowel Dis 2001;7:348-349.

4 Galiatsatos P, Shrier I, Lamoureux E, Szilagyi A: Meta-analysis of outcome of cytomegalovirus colitis in immunocompetent hosts. Dig Dis Sci 2005;50:609-616.

5 Knosel T, Schewe C, Dietel M, Petersen I: Cytomegalovirus is not associated with progression and metastasis of colorectal cancer. Cancer Lett 2004;211:243-247. 


\section{Case Reports in Oncology}

\begin{tabular}{l|l}
\hline Case Rep Oncol 2013;6:109-113 & \\
\hline DOI: $\underline{10.1159 / 000348711}$ & $\begin{array}{l}\text { ○ 2013 S. Karger AG, Basel } \\
\text { www.karger.com/cro }\end{array}$ \\
\hline
\end{tabular}

Saito et al.: Hemorrhagic Cytomegalovirus Colitis in a Postoperative Colon Cancer Patient

-6 Akintola-Ogunremi O, Luo Q, He TC, Wang HL: Is cytomegalovirus associated with human colorectal tumorigenesis? Am J Clin Pathol 2005;123:244-249.

7 Biron KK: Antiviral drugs for cytomegalovirus diseases. Antiviral Res 2006;71:154-163.

-8 Ahmed A: Antiviral treatment of cytomegalovirus infection. Infect Disord Drug Targets 2011;11:475-503.

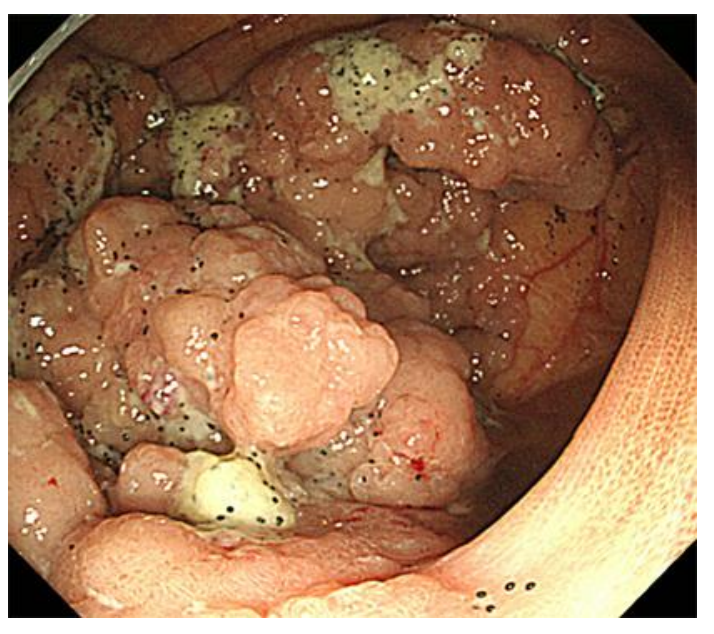

Fig. 1. Colonoscopy revealed a laterally spreading tumor of the granular type at the cecum.

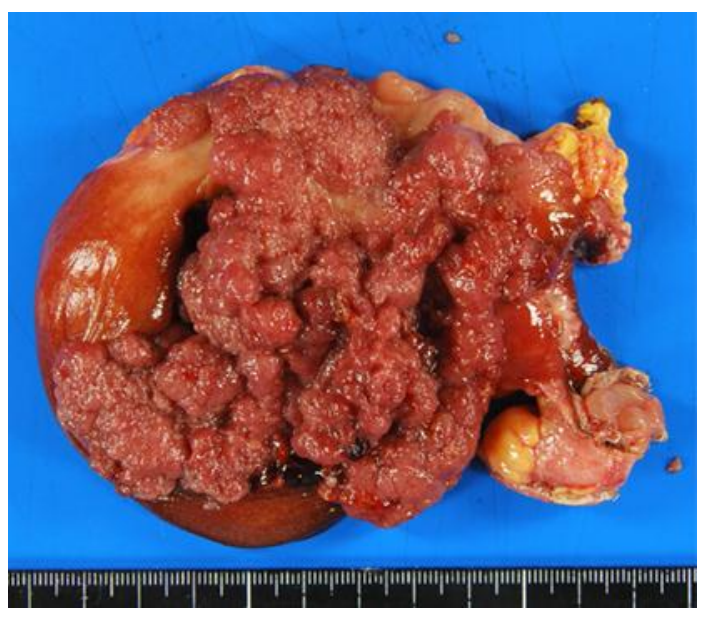

Fig. 2. Resected specimens of the tumor. Histopathological examination revealed papillary adenocarcinoma in villotubular adenoma. 


\section{Case Reports in Oncology}

Case Rep Oncol 2013;6:109-113

DOI: $\underline{10.1159 / 000348711}$

Saito et al.: Hemorrhagic Cytomegalovirus Colitis in a Postoperative Colon Cancer Patient
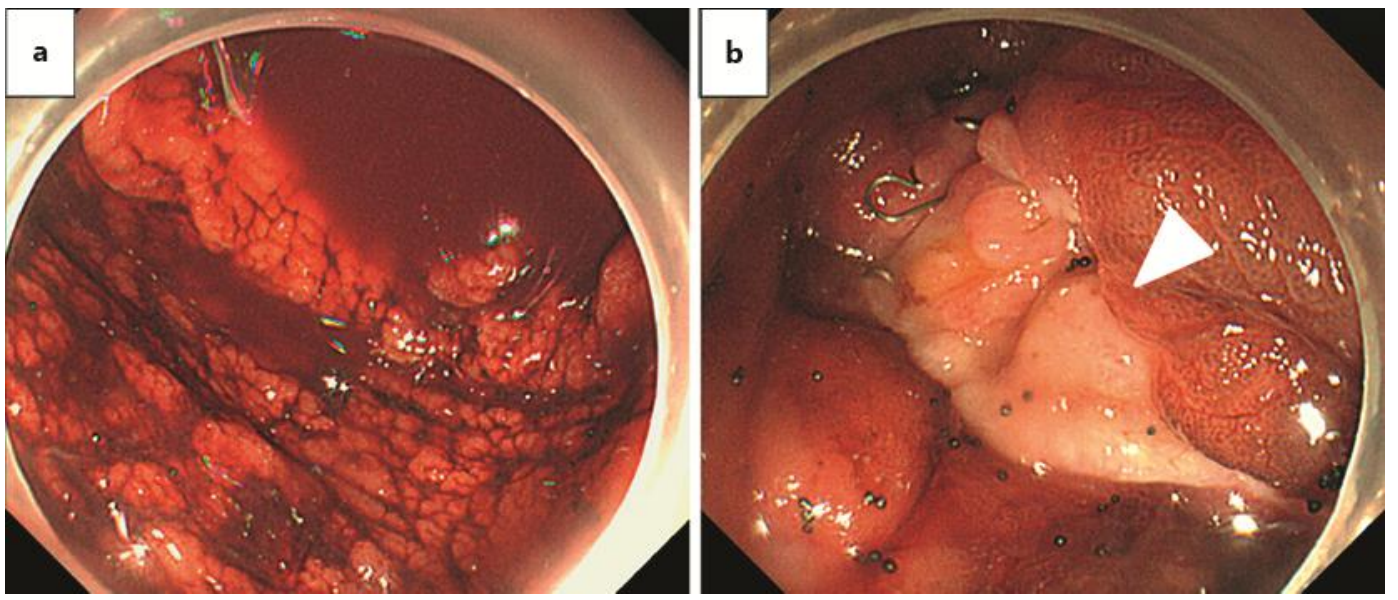

Fig. 3. Colonoscopy revealed bleeding (a) and the longitudinal ulcer at the anastomosis (b, white arrowhead). 